\title{
Antibody structure
}

\author{
SYDNEY COHEN
}

From the Department of Chemical Pathology, Guy's Hospital Medical School, London

A consideration of the overall properties of antibodies has for some time suggested that their structural features must be unique among protein molecules. In the first place, serum antibodies within a species share a variety of physical, chemical and antigenic properties indicating that all have a common basic structure. However, the immunoglobulins of all animal species investigated occur in several forms, somewhat arbitrarily designated as classes, subclasses, or types, and distinguished on the basis of their chemical and biological properties. In addition, the great diversity of antigens which evoke an immune response and the narrowly defined specificity of the serum antibodies formed indicate that each individual can synthesize a very large number of antibodies-probably more than $10^{5}$ and there is conclusive evidence that these differ from one another in their covalent structure. The fundamental problem of antibody structure therefore concerns the nature of the molecular modifications which are superimposed upon a relatively constant basic configuration to generate a finite number of immunoglobulin classes and a very large assortment of distinct combining specificities. The extent to which this problem has been elucidated by studies of protein structure is outlined in this paper. It has been reviewed by Leslie and Cohen (1973).

There is an additional intriguing problem which concerns the way that antibodies mediate complex biological reactions including, for example, the expression of acquired protective immunity or immediate hypersensitivity. There are very few instances in which the interaction of antigen with antibody leads directly to biological effects. Such primary reactions are limited to the inactivation of enzymes and toxins and the neutralization of some viruses and protozoa. In the great majority of cases the biological expression of immune reactivity requires interaction of antibody with either the complement system or with specific cell surfaces. Such reactions involve specific effector sites on the antibodies which are distributed unevenly among the classes and subclasses of immunoglobulin. These sites are involved in complement- or cell-mediated lysis, opsonization by macrophages and mast cell degranulation resulting in immediate hypersensitivity. None of these reactions is mediated by antibody alone but all can be initiated by the combination of antibody with antigen. How do antigens trigger these events and can their effects be explained in terms of a structural modification of antibody molecules?

\section{Structure of Immunoglobulins (Ig)}

The four-chain structure of Ig, consisting of two heavy and two light chains covalently linked by interchain disulphide bonds (fig 1), was originally

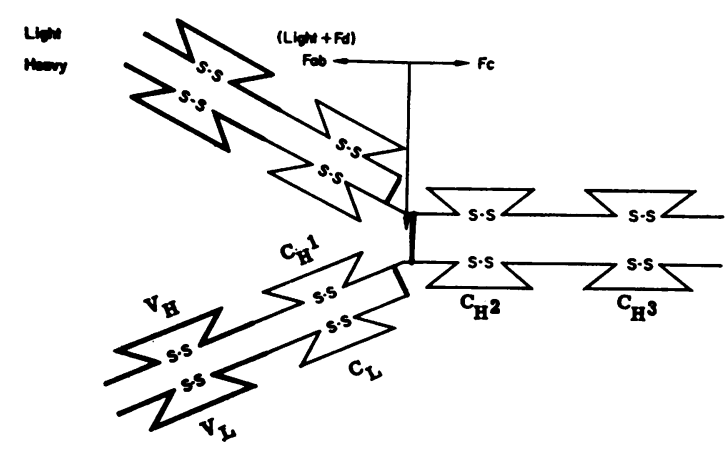

Fig 1 Diagrammatic representation of the IgG molecule made up of two heavy and two light chains. Intrachain disulphide bonds enclosing loops of 60-70 amino acids occur along the length of heavy and light chains. Heavy lines denote the $N$-terminal variable stretches of heavy $\left(V_{\mathrm{H}}\right)$ and light $\left(V_{\mathrm{L}}\right)$ chains. The vertical arrow shows the site of papain digestion which splits the molecule within the hinge region of the heavy chain to give Fab and Fc fragments.

proposed in 1962 by Porter for rabbit IgG, and is now known to apply to antibodies of all vertebrate species studied including primitive elasmobranch fish. The various classes and subclasses of $\mathrm{Ig}$ within a single species are differentiated on the basis of their heavy chains (table I). High molecular weight antibodies, such as serum IgM and seromucous 


\begin{tabular}{|c|c|c|c|c|c|}
\hline \multirow[t]{2}{*}{ Property } & \multicolumn{5}{|c|}{ Immunoglobulin } \\
\hline & $\operatorname{Ig} G$ & $\operatorname{Ig} A$ & $\operatorname{Ig} M$ & $\operatorname{Ig} D$ & $I g E$ \\
\hline $\begin{array}{l}\text { Structural } \\
\text { Molecular weight }\end{array}$ & 150000 & $\begin{array}{l}160000^{1} \\
\text { (serum) } \\
370000 \\
\text { (secretory) }\end{array}$ & 900000 & 170000 & 185000 \\
\hline \multicolumn{6}{|l|}{ Heavy chains } \\
\hline $\begin{array}{l}\text { Classes } \\
\text { Subclasses } \\
\text { Percentage in serum }\end{array}$ & 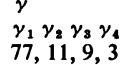 & $\begin{array}{l}a \\
a_{1} a_{2} \\
90,10\end{array}$ & $\begin{array}{l}\mu \\
\mu_{1} \mu_{2}\end{array}$ & d & $\epsilon$ \\
\hline Carbohydrate $(\%)$ & $2 \cdot 9$ & $7 \cdot 5$ & $11 \cdot 8$ & $11 \cdot 3$ & $12 \cdot 1$ \\
\hline $\begin{array}{l}\text { Light chains } \\
\text { J chains }\end{array}$ & $\kappa, \lambda$ & $\begin{array}{c}\kappa, \lambda \\
+\end{array}$ & $\begin{array}{c}\kappa, \lambda \\
+\end{array}$ & $\kappa, \lambda$ & $\kappa, \lambda$ \\
\hline \multicolumn{6}{|l|}{ Biological } \\
\hline Antibody activity & + & + & + & + & + \\
\hline C fixation & $+\gamma_{1} \gamma_{2} \gamma_{3}$ & 0 & $+\mu_{1}$ & 0 & 0 \\
\hline Macrophage attachment & $+\gamma_{1} \gamma_{3}$ & & + & & 0 \\
\hline Placental transfer & $+\gamma_{1} \gamma_{3} \gamma_{4}$ & 0 & 0 & & 0 \\
\hline \multirow{2}{*}{\multicolumn{6}{|c|}{$\begin{array}{l}\text { Seromucous secretion } \\
\text { Tissue sensitization }\end{array}$}} \\
\hline $\begin{array}{l}\text { Tissue sensitization } \\
\text { Homologous spp }\end{array}$ & & & & & + \\
\hline Heterologous spp & $+\gamma_{1} \gamma_{2} \gamma_{3}$ & 0 & 0 & $\mathbf{0}$ & $0^{2}$ \\
\hline Combine with Staph a p:otein & $+\gamma_{1} \gamma_{4} \gamma_{4}$ & 0 & $\mathbf{0}$ & 0 & 0 \\
\hline
\end{tabular}

$\operatorname{IgA}$, consist of polymers of the four-chain unit linked by a J-chain, which is rich in half-cysteine residues and common to both classes. Antibodies of all classes have light chains of two types $(\kappa$ or $\lambda)$.

Certain features of this structure are of particular significance in regard to the generation of antibody specificity and the properties of immunoglobulin effector sites.

Ig chains contain an $\mathrm{N}$-terminal variable region (V) comprising about 120 amino acid residues in which all heterogeneity within a subclass is localized. This was demonstrated first by Hilschmann and Craig in 1965 for human $\kappa$-chains, but V-regions of similar extent occur on all light and heavy chains.

The remaining C-terminal portions (C) of $\mathrm{Ig}$ chains within a given subclass have, by contrast, an invariant structure apart from differences attributable to individual genotypes. The complete sequence of a human $\gamma_{1}$-chain was elucidated by Edelman (1971) and considerable information is available for C-regions of other $\gamma$-subclasses and for the $\mu-, \alpha-$, and $\epsilon$-chains. Available data reveal considerable sequence differences between heavy chain classes, eg, between $\gamma_{1^{-}}$and $\mu$-chains while subclasses show far greater homology.

All Ig chains show a periodic arrangement of intrachain disulphide bonds (fig 1) dividing light chains into two domains $\left(V_{L}\right.$ and $\left.C_{L}\right), \gamma$ and $\alpha$-chains into four domains $\left(V_{H} 1, C_{H} 1, C_{H} 2\right.$, and $\left.C_{H} 3\right)$ and $\mu$ - and $\epsilon$-chains into five domains. $V_{L}$ and $V_{H}$ show homology with one another but not obviously with
$\mathrm{C}$ regions and are associated with antigen-binding sites. The variable regions of light chains $\left(V_{\kappa}\right.$ and $\left.V \lambda\right)$ can be unambiguously differentiated, but variable regions of heavy chains $\left(\mathrm{V}_{\mathrm{H}}\right)$ appear common to alk classes. $\mathbf{C}_{\mathbf{H}}$ regions carry the biologically active sites which are variously distributed on the classes andê subclasses of Ig (table I) (reviewed by Spiegelberg 1974).

The heavy chain region which contains the Cys residues involved in interchain disulphide bonding shows considerable variation between classes and subclasses and no homology with other sections of the heavy or light chain. This stretch of the $\gamma$ - and $\alpha$-chain is rich in proline and is susceptible to proteolysis. This section, on the basis of electrong micrography and other physical measurements, has been designated the hinge region (fig 1) but the extent to which the Fab arms can move relative tô one another remains uncertain (Metzger, 1974).

\section{Antibody-combining Sites}

Physicochemical studies of antibody saturated witb antigen have indicated that there are two discrete combining sites per $7 \mathrm{~S}$ molecule and the subsequently? established monovalency of Fab fragments ando symmetrical chain structure are consistent with thiso The valency of pentameric $\operatorname{IgM}\left(\mathrm{H}_{10} \mathrm{~L}_{10}\right)$ has proved variable, but it now seems that IgM has 10 site and that binding of larger antigens may reduce the effective valency to five sites per antibody molecule 
Similarly, polymeric IgA appears to have four combining sites.

The size of the antibody-combining sites, assessed by determining the dimensions of antigenic determinants which give optimum binding, has been estimated as $3.4 \mathrm{~nm} \times 1.7 \mathrm{~nm} \times 0.6 \mathrm{~nm}$. This indicates that 10 to 20 amino acids comprise the combining site and make direct contact with the antigenic determinant. Electron microscopy of antibodies complexed with a divalent hapten indicates that the binding site is situated at the extreme ends of the Fab regions and has a maximum depth of about $4.0 \mathrm{~nm}$.

In general, antibodies react most strongly with the antigen which elicited their synthesis and show graded affinities for structurally related compounds. Stereo-complementarity of the antigenic determinant and the antibody-combining site is thought to provide the major contribution to the non-covalent associations which determine specificity. It is therefore surprising that high affinity antibodies may show strong cross reactions with haptens of quite distinct structure. This suggests that whereas weak hydrophobic interactions between antigen and a closely fitting antibody site may be predominant in low affinity antibodies, additional stronger forces with less stereospecificity may operate in high affinity antibodies; their nature would presumably vary with the chemical identity of functional groups in the antigenic determinant. Another explanation of unexpected cross specificities is that the antibody cavity may contain more than one binding site and that distinct small haptens might adhere to separate sites on opposite walls of the cavity, and that steric hindrance accounts for competitive inhibition of one hapten by the other.

Direct evidence that antibody specificity is determined by amino acid sequence arose from a study in which complete reduction and unfolding of Fab fragments produced concomitant loss of antibody activity, while removal of the denaturant was associated with significant recovery of the original activity. This indicates that the chain folding which generates combining specificity is dependent on the primary sequence of the Fab fragments. The presence of variable sequences confined to the $\mathrm{N}$ terminal portions of Fab fragments again suggested that primary structure generates combining specificities. Analysis of the variable sequences in antibodies of defined specificity has been hampered by the heterogeneity of most purified antibodies raised against even the simplest hapten and also by the rarity of myeloma proteins having specific binding affinities within the range for elicited antibodies. Comparative sequence studies on monoclonal proteins have revealed, within the V-regions of both heavy and light chains, three short stretches with high sequence variability (Wu and Kabat, 1970). Hypervariable regions in both $\mathrm{H}$ and $\mathrm{L}$ chains are brought into close proximity by chain folding and by the $\mathrm{N}$-terminal intrachain disulphide bridge. The location of combining sites has been sought by employing hapten determinants attached to a chemical group which will interact covalently with residues in or near to the active site. This technique of affinity labelling has provided results consistent with involvement of hypervariable regions from both light and heavy chains in forming the combining cavity.

Conclusive evidence on the structure of the active site is being obtained by $x$-ray crystallography of purified, homogeneous Fab fragments. The $0.28 \mathrm{~nm}$ resolution of such a fragment from a myeloma protein has provided a three-dimensional model of Fab. This contains four globular subunits corresponding with the variable $\left(V_{H}\right.$ and $\left.V_{L}\right)$ and constant $\left(C_{H} 1\right.$ and $C_{L}$ ) domains of Fab. The hypervariable regions of light and heavy chains occur in close proximity at one end of the molecule and enclose a cavity which probably represents the combining site (Poljak, Amzel, Avey, Chen, Phizackerley, and Saul, 1973). Further studies have revealed that a hapten having specific affinity for this Fab fragment is indeed bound in the shallow groove between heavy and light chains and appears to be in contact with segments of the Ig sequence which contain the hypervariable regions (Amzel, Poljak, Saul, Varga, and Richards, 1974).

\section{Ig Effector Sites}

The IgG Fc fragment (molecular weight 50000 ) prepared by papain hydrolysis retains many active sites of the original molecule, including the capacity to fix complement, attach to mast cells and macrophages, combine with staphylococcal protein A, regulate IgG catabolic rate, and cross the placental membrane and gut wall of the newborn (table II). The sites responsible for these properties are therefore contained within the two C-terminal intrachain disulphide loops of the $\gamma$-chain $\left(\mathrm{C}_{\mathrm{H}} 2\right.$ and $\mathrm{C}_{\mathrm{H}} 3$, figs 1 and 2). Smaller fragments of Fc, referred to as $\mathrm{Fc}^{\prime}$ or $\mathrm{pFc}^{\prime}$, which comprise only the C-terminal domain $\left(\mathrm{C}_{\mathrm{H}} 3\right)$ can be prepared by hydrolysis with pepsin or papain (fig 2). These fragments carry some isotypic and allotypic determinants, combine with rheumatoid factors of certain specificities, and may retain activity for macrophage and $B$ cell attachment (table II). Evidence has been obtained for complement-fixing activity in the $\mathrm{C}_{\mathrm{H}} 2$ domain.

Certain Ig effector sites appear to be localized in 


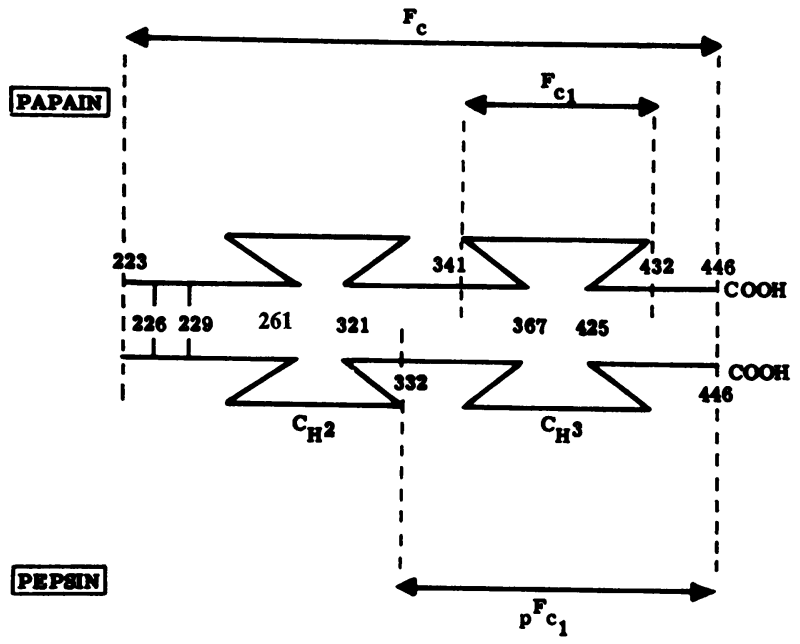

Fig 2 Diagrammatic representation of the $F c$ fragment of human $I g G_{1}$ containing the $C_{\mathrm{H}_{2}}$ and $\overline{\bar{T}}$ $\mathrm{CH}_{3}$ domains ( $f$ ig $\mathrm{I}$ ) and showing sites of cleavage by papain and pepsin to yield $F^{\prime}$ and $p F c^{\prime}$ pieces containing the $\mathrm{CH}_{3}$ domain.

Residues are numbered from the $\mathbf{N}$-terminus; numbers in the centre refer to cystine residues.

the $\left(\mathrm{Fab}^{\prime}\right)_{2}{ }^{1}$ fragment, possibly within the $\mathrm{C}_{\mathrm{H}} 1$ domain (fig 1). Guinea pig $\mathrm{IgG}_{1}{ }^{2}$ fails to fix complement in the presence of antigen or lyse antigencoated cells in the presence of complement. However, washed specific precipitates containing IgG $_{1}$ antibodies do fix complement by an alternate pathway beginning with $\mathrm{C} 3$, the third component of complement. The sites which fix $\mathrm{Clq}^{3}$ for conventional complement activation are present on the $\mathrm{Fc}$

\begin{tabular}{lllll}
\hline Activity & $F c$ & & $F c^{\prime}$ & \\
\cline { 2 - 3 } & $\left(C_{\mathrm{H} 2} 2 C_{\mathrm{H}} 3\right)$ & & $\left(C_{\mathrm{H}} 3\right)$ & $\left(C_{\mathrm{H}} 2\right)$ \\
\hline Binding of: & & & \\
$\quad$ Macrophages & + &,+ & \\
B-lymphocytes & + & + & \\
K-cells & + & - & \\
Complement & + & & + \\
$\begin{array}{l}\text { Rheumatoid factor } \\
\text { Staph a protein }\end{array}$ & + & & \\
Membrane transmission & + & & \\
Catabolism control & + & & \\
\hline
\end{tabular}

Table II Biological activities of Fc fragment

fragment of $\mathrm{IgG}_{2}$ but absent from $\mathrm{IgG}_{1}$, whereas those which initiate the $\mathrm{C} 3$ shunt mechanism are present on $\left(\mathrm{Fab}^{\prime}\right)_{2}$ fragments from guinea pig $\mathrm{IgG}_{1}$ and $\mathrm{IgG}_{2}$ and also on human IgA. Localization of biologically active sites in other classes of Ig has not been thoroughly studied. However, the Fc fragment of IgE contains the sites responsible for the attachment to isologous tissues while its C-

${ }^{1}\left(\mathrm{Fab}^{\prime}\right)_{2}$ is a fragment produced by pepsin which splits the molecule on the $\mathrm{C}$-terminal side of the interheavy chain disulphide bridge (see fig 1). When the latter is also split two Fab' fragments result. 'The subclass of IgG containing $\gamma_{1}$ heavy chains (see table I)

'One of the three subunits of the first component of complement, $\mathrm{C} 1$. terminal $\mathrm{Fc}^{\prime}$ fragment is inactive in this regard. The Fc fragment of human IgA has not been isolated, $\vec{\theta}$ but mouse and rabbit IgA Fc can be prepared and, from the latter species, binds the secretory component characteristically associated with seromucous IgA.

The failure to isolate active fragments from the Fc portions of Ig molecules has meant that the structure of the effector sites remains obscure. The IgG sites which react with $\mathrm{Clq}$ and bind to macro-o응 phages and the IgE sites which attach to mast cells are all inactivated by mild reduction suggesting that integrity of interheavy chain disulphide bonds in the hinge region is essential.

Available evidence suggests that where the sameo biological activity is mediated by different Ig classes, the sites involved are structurally distinct. Foro example, mouse 7S and 19S antibodies both bind 3 to macrophages but not competitively, and only the은 19S binding is dependent on the presence of $\mathrm{Ca}^{++}$ ions. In other instances, different Ig classes may bind을. the same receptor, but with different affinities, indicating that their reactive sites are structurallyo distinct. For example, the affinity of $\mathrm{Clq}$ is greatest ${ }^{N}$ for $I_{g G}$ and progressively less for $\operatorname{IgG}_{1}$ and $I_{g G}$. స్ట

\section{Triggering of Ig-mediated Biological Reactions}

Certain biological activities are mediated by single $\stackrel{\oplus}{+}$ Ig molecules, eg, the control of Ig catabolic rate, 0 transfer across placental and gut membranes, and $\overrightarrow{0}$ combination with staphylococcal protein A (fig 3). However, other reactions, including immediate hypersensitivity, complement activation and opsonization by macrophages are not initiated by 
A

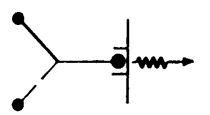

Placental transfer

Gastro-intestinal transfer

Control catabolic rate

Combination staph aureus protein A

B

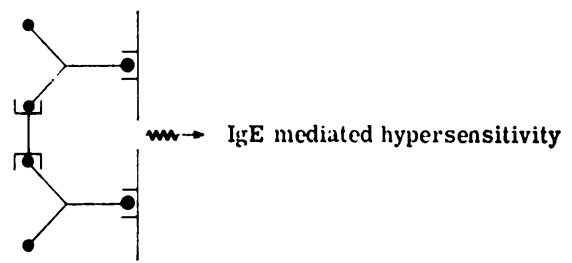

C

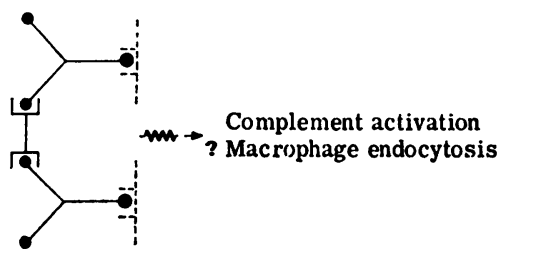

Fig 3 Ig-mediated effector mechanisms. A Sites with biological activity on single antibody molecules. B and $\mathrm{C}$ High-and low-affinity sites on free antibodies which can combine with acceptor sites but require cross linkage to an adjacent antibody molecule to become biologically active.

individual Ig molecules, but only by more than one antibody linked by polyvalent antigen (fig 3, $B$ and $C$ ). These reactions therefore require an antigen-dependent interaction between $\mathrm{Fab}$ and $\mathrm{Fc}$ sections of Ig molecules (reviewed by Metzger, 1974).

\section{Antigen as a Trigger for Antibody-mediated Reactions}

IgM and IgG antibodies in free solution combine reversibly with the first component of complement (C1), but this does not initiate the subsequent steps of complement activation. In the case of antigen attached to cell surfaces, the interaction with $\mathrm{C1}$ by two adjacent IgG molecules fixed to the cell antigens is sufficient to initiate the full cycle of lysis. Antigen density on the cell surface is therefore a critical factor in determining the occurrence of complement-mediated cytotoxic reactions (fig 4). Thus, the non-lytic nature of anti-Rh antibodies is attributed to the comparative paucity of $D$ antigen sites on the erythrocyte surface. Similarly, the adsorption of host components onto cell surfaces may alter the antigen density of established tumours and parasites and permit evasion of complementmediated cytolysis, which nevertheless remains effective against homologous cells newly introduced

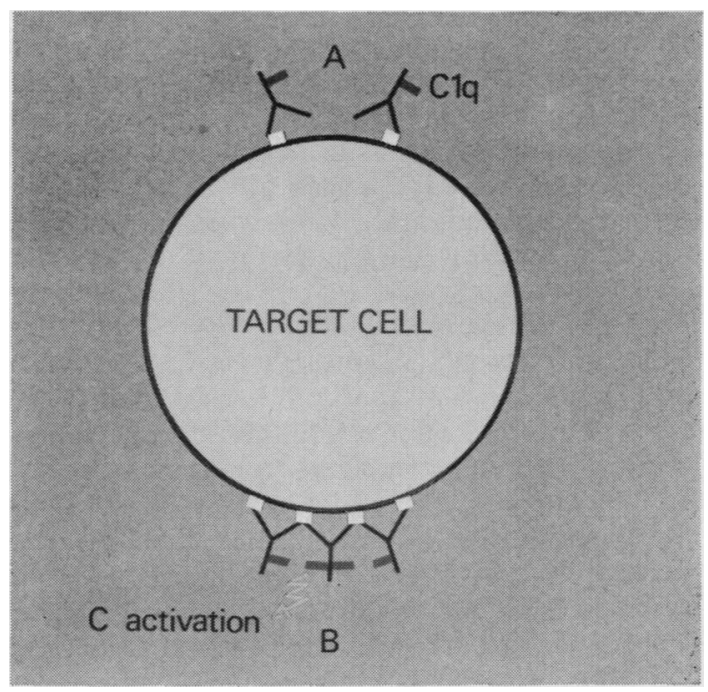

Fig 4A Wide separation of the antigenic determinants and the reactive IgG molecules on a cell surface with consequent failure to initiate complement activation.

Fig 4B Cross-linked IgG molecules on a cell surface initiate complement-mediated lysis.

into the host-a phenomenon referred to as concomitant immunity.

Mast cells have homocytotrophic antibodies (IgE) bound to their surface membranes (fig $5 \mathrm{~A}$ ) but are triggered for histamine release only after the bound molecules have become cross linked, usually by polyvalent antigen (fig 5B). By contrast, the majority of monovalent haptens are totally ineffective (fig 5D). The requirement for cross linking implies that the density of cell-bound IgE of given specificity is a critical factor determining antigen-induced hyper-

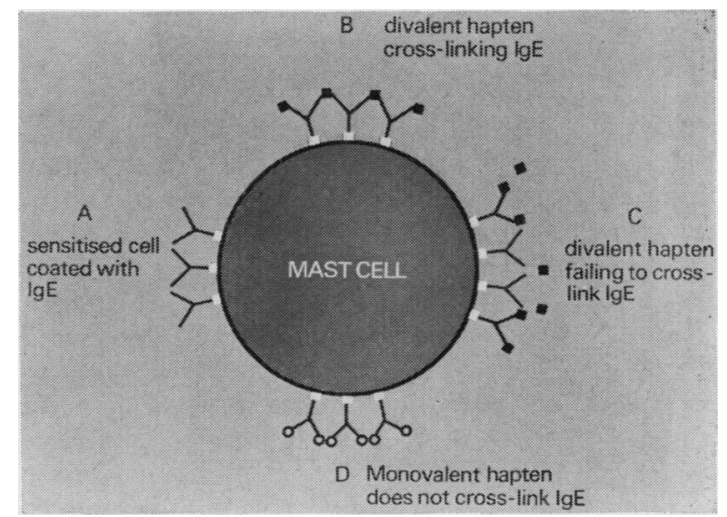

Fig 5 Diagram illustrating the requirements for IgEmediated hypersensitivity reactions (see text). 
sensitivity (fig $5 \mathrm{C}$ ). The nature of the cross linkage between IgE molecules appears unimportant since multivalent antigen and divalent anti-IgE antibodies are both potent initiators, whereas monovalent antigens or Fab fragments from anti-IgE antibodies are ineffective. Similarly, chemically aggregated IgE directly induces immediate hypersensitivity reactions in human subjects and guinea pigs, though the dosage required is greater than that needed with the other cross-linking mechanisms.

Studies of cytophilic antibody have shown that native Ig molecules of appropriate class bind to the surface of macrophages; in addition, the attachment of antigen-antibody complexes to macrophages can be inhibited by excess free IgG. These observations indicate that cytophilic sites, at least on $\mathrm{IgG}$, are available on the surface of native molecules. The amount of antibody bound to macrophages and the affinity of binding are increased by cross linking with polyvalent antigen at equivalence, but are unaffected by monovalent or excess polyvalent antigen. Particles such as red blood cells, after reaction with cytophilic antibody, become attached to macrophages and remain bound to the surface if left at room temperature or $4^{\circ} \mathrm{C}$. Incubation of such preparations at $37^{\circ}$ causes phagocytosis of all attached red cells. Phagocytosis occurs without complement when the opsonizing antibody is IgG but complement components are required for ingestion of membrane-bound particles coated with IgM. Whether cross linking of IgG by antigen is a required trigger for phagocytosis is unknown, since the influence of antigenic valency upon endocytosis has not been evaluated.

There appear to be two ways in which cross linking of antibody could initiate biological reactions which involve sites on Fc sections of Ig molecules.

IG CONFORMATIONAL CHANGE

Combination with antigen could expose or generate biologically active sites whose affinity is not fully expressed in the native molecule; combination with appropriate receptors would then trigger the biological event. This sequence would seem likely if native Ig did not bind receptors and did not inhibit binding by antigen-antibody complexes. In fact, as outlined above, the sites for $\mathrm{Cl}$ fixation, mast cell attachment and macrophage binding are all active in free Ig of the appropriate class; it remains possible that the binding affinity of $\mathrm{Ig}$ sites is increased or new sites are exposed by an antigendependent conformational change and that this triggers the receptor to initiate the biological sequence. However, physico-chemical studies of immunoglobulins in solution provide little evidence for antigen-induced conformational changes (Metzger, 1974; Leslie and Cohen, 1973).
LATTICE FORMATION

The reactivity of effector sites on native $\mathrm{Ig}$ molecules $\vec{\Rightarrow}$ and the failure to demonstrate convincingly antigen- $\stackrel{\rho}{\longrightarrow}$ mediated conformational changes in antibody molecules leave open the possibility that biological 흔 effects are induced by lattice formation which follows $\frac{\pi}{\sigma}$

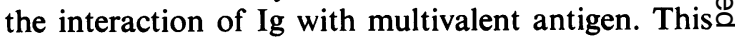
could occur where the Fc regions of free antibodies have low affinity binding sites, eg, for Clq or for $\vec{\circ}$ macrophage receptors, so that antigen-antibodycomplexes have increased functional affinity by $\vec{\omega}$ virtue of the number of sites in close proximity. In as multicomponent system (such as the complemento system) the increased functional affinity of the $\frac{\text { s }}{-}$ complex could cause retention of the first componento for sufficient time to allow for activation and inter-. action with further components, thereby initiating ${ }_{0}$ the full sequence of the response. A similar mechan-? ism seems possible for antibody-induced phagocyto- $\bar{c}$ sis by macrophages.

The influence of multivalency upon overall affinity is illustrated by the fact that free IgG and the 7S subunit of IgM bind similar amounts of $\mathrm{Clq} \overrightarrow{\mathrm{O}}$ while pentameric IgM, which presumably containsor five complement-fixation sites per molecule, is 150 times more active. Larger aggregates of IgG and IgM bind up to 100 times more $\mathrm{Clq}$ on a weight basis. These data correlate with the observation thato two or more IgG molecules in close proximity onष्ठ the cell surface are required to produce a lytic lesion whereas a single IgM molecule is sufficient to produce the same effect.

The mechanism of mast cell degranulation medi ated by IgE is of interest since the native antibody has. a high affinity for cell surface receptors and yefo stimulates histamine release only after cross-linking The trigger may involve conformational changes within the antibody which generate either additiona $B$ binding activity or conformational changes within receptors at the cell surface. However, it is simpler to propose that lattice formation between bound IgED molecules leads to aggregation of cell surface receptors and this provides the trigger for mast celi degranulation (fig 5).

\section{Conclusions}

Antibody molecules show a high degree of individuab antigen-binding specificity but may share certair吊 biological properties, such as the capacity to fix? complement, which are characteristic of the class of Ig to which they belong. This functional dualism i⿱尺्乛龰 generated by peptide chains which are unique i⿱ having variable $\mathrm{N}$-terminal (V) regions and constang C-terminal (C) regions.

The two antigen-binding sites of each four-chaip 
Ig molecule are contained within the V-regions of both heavy and light chains. Specificity is determined by primary structure. $X$-ray diffraction studies show that combining sites are generated by the short hypervariable stretches in heavy and light chains. The stereochemical configuration of the antibody site is regarded as the prime determinant of specificity.

Biological effector sites occur predominantly in the Fc portion of the molecule which comprises the two C-terminal intrachain disulphide loops of the heavy chains. Little information is available about the location, chemical structure, specificity, or affinity of these sites. All appear accessible in native Ig and some, including those responsible for placental and gastrointestinal transfer of Ig molecules and for the concentration-dependent control of IgG catabolic rate, are active in free molecules. Other effector sites are present on free Ig molecules and interact with their relevant receptors, but the full biological sequence is initiated only after the bound antibody has become cross linked, usually by polyvalent antigen. This is true for complement activation, IgE-mediated hypersensitivity and possibly also for opsonin-induced phagocytosis.

The cross linkage of Ig leads to lattice formation and this may trigger the biological sequence by producing aggregation or conformational change in the receptor. An Ig lattice signal is consistent with the known presence of effector sites on native $\mathrm{Ig}$ molecules, the failure of monovalent or excess polyvalent antigen to induce reactions, and the triggering which occurs with diverse forms of cross linking. Ig conformational changes may have a role in initiating biological reactions, but their occurrence does not seem mandatory.

\section{References}

Amzel, L. M., Poljak, R. J., Saul, F., Varga, J. M., and Richards, F. F. (1974). The three-dimensional structure of a combining regionligand complex of immunoglobulins NEW at 3.5 A resolution. Proc. nat. Acad. Sci. (Wash.), 71, 1427.

Edelman, G. M. (1971). Antibody structure and molecular immunology. Ann. N.Y. Acad. Sci., 190, 5.

Hilschmann, N., and Craig, L. C. (1965). Amino acid sequence studies with Bence Jones proteins. Proc. nat. Acad. Sci. (Wash.), $53,1403$.

Leslie, R. G. Q., and Cohen, S. (1973). The active sites of immunoglobulin molecules. In Essays in Fundamental Immunology, I, edited by I. Roitt, p. 1. Blackwell, Oxford.

Metzger, H. (1974). Effect of antigen binding in the properties of antibody. Advanc. Immunol., 18, 169.

Poljak, R. J. Amzel, L. M., Avey, H. P., Chen, B. L., Phizackerley, R. P., and Saul, F. (1973). Three-dimensional structure of the Fab fragment of a human immunoglobulin at $2.8 \AA$ resolution. Proc. nat. Acad. Sci. (Wash.), 70, 3305.

Porter, R. R. (1962). In Basic Problems of Neoplastic Disease, edited by A. Gellhorn and E. Hirschberg, p. 177. Columbia University Press, New York.

Spiegelberg, H. L. (1974). Biological activities of immunoglobulins of different classes and subclasses. Advanc. Immunol., 19, 259.

Wu, T. T., and Kabat, E. A. (1970). An analysis of the sequences of the variable regions of Bence Jones proteins and myeloma light chains and their implications for antibody complementarity.J exp. Med., 132, 211. 